\title{
NEW STABILITY CONDITIONS FOR POSITIVE CONTINUOUS-DISCRETE 2D LINEAR SYSTEMS
}

\author{
TADEUSZ KACZOREK
}

Faculty of Electrical Engineering

Białystok Technical University, ul. Wiejska 45D, 15-351 Białystok, Poland

e-mail:kaczorek@isep.pw.edu.pl

\begin{abstract}
New necessary and sufficient conditions for asymptotic stability of positive continuous-discrete 2D linear systems are established. Necessary conditions for the stability are also given. The stability tests are demonstrated on numerical examples.
\end{abstract}

Keywords: positive systems, 2D linear systems, continuous-discrete systems.

\section{Introduction}

In positive systems inputs, state variables and outputs take only nonnegative values. A variety of models having positive systems behavior can be found in engineering, management science, economics, social sciences, biology and medicine, etc. An overview of the state of the art in positive systems is given in the monographs of Farina and Rinaldi (2000) as well as Kaczorek (2002).

Positive continuous-discrete 2D linear systems were introduced by Kaczorek (1998) along with positive hybrid linear systems (Kaczorek, 2007) and positive fractional 2D hybrid systems (Kaczorek, 2008a). Various methods of solvability of 2D hybrid linear systems were discussed by Kaczorek et al. (2008), and the solution to singular 2D hybrids linear systems was derived by Sajewski (2009). The realization problem for positive $2 \mathrm{D}$ hybrid systems was addressed by Kaczorek (2008b). Some problems of dynamics and control of 2D hybrid systems were considered by Dymkov et al. (2004) and Gałkowski et al. (2003). The problems of stability and robust stability of 2D continuous-discrete linear systems were investigated by Bistritz (2003), Busłowicz (2010a; 2010b, 2011) and Xiao (2001a; 2001b; 2003). Recently, stability and robust stability of a general model and of a Roesser type model of scalar continuous-discrete linear systems were analyzed by Busłowicz (2010a; 2010b; 2011).

In this paper, new necessary and sufficient conditions for asymptotic stability of positive continuous-discrete 2D linear systems will be presented.

The following notation will be used: $\mathbb{R}$ is the set of real numbers, $\mathbb{Z}_{+}$stands for the set of nonnegative inte- gers, $\mathbb{R}^{n \times m}$ denotes the set of $n \times m$ real matrices, $\mathbb{R}_{+}^{n \times m}$ is the set of $n \times m$ matrices with nonnegative entries and $\mathbb{R}_{+}^{n}=\mathbb{R}_{+}^{n \times 1}, I_{n}$ denotes the $n \times n$ identity matrix.

\section{Preliminaries}

Consider the linear autonomous continuous-discrete 2D system (Kaczorek, 1998; 2002)

$$
\begin{array}{r}
\dot{x}(t, i+1)=A_{0} x(t, i)+A_{1} \dot{x}(t, i)+A_{2} x(t, i+1), \\
t \in \mathbb{R}_{+}, \quad i \in \mathbb{Z}_{+},
\end{array}
$$

where $\dot{x}(t, i)=\partial x(t, i) / \partial t, x(t, i) \in \mathbb{R}^{n}, A_{k} \in \mathbb{R}^{n \times n}$ for $k=0,1,2$.

Definition 1. The linear continuous-discrete 2D system (1) is called (internally) positive if $x(t, i) \in \mathbb{R}_{+}^{n}, t \in \mathbb{R}_{+}$, $i \in Z_{+}$for all initial conditions

$$
\begin{aligned}
x(0, i) \in \mathbb{R}_{+}^{n}, \quad i & \in \mathbb{Z}_{+}, \\
x(t, 0) & \in \mathbb{R}_{+}^{n}, \dot{x}(t, 0) \in \mathbb{R}_{+}^{n}, \quad t \in \mathbb{R}_{+} .
\end{aligned}
$$

Theorem 1. (Kaczorek, 1998; 2002) The linear continuous-discrete $2 D$ system (1) is positive if and only if

$$
\begin{aligned}
A_{2} \in M_{n}, \quad A_{0}, A_{1} \in \mathbb{R}_{+}^{n \times n}, & \\
& A_{0}+A_{1} A_{2} \in \mathbb{R}_{+}^{n \times n},
\end{aligned}
$$

where $M_{n}$ is the set of $n \times n$ Metzler matrices (with nonnegative off-diagonal entries). 


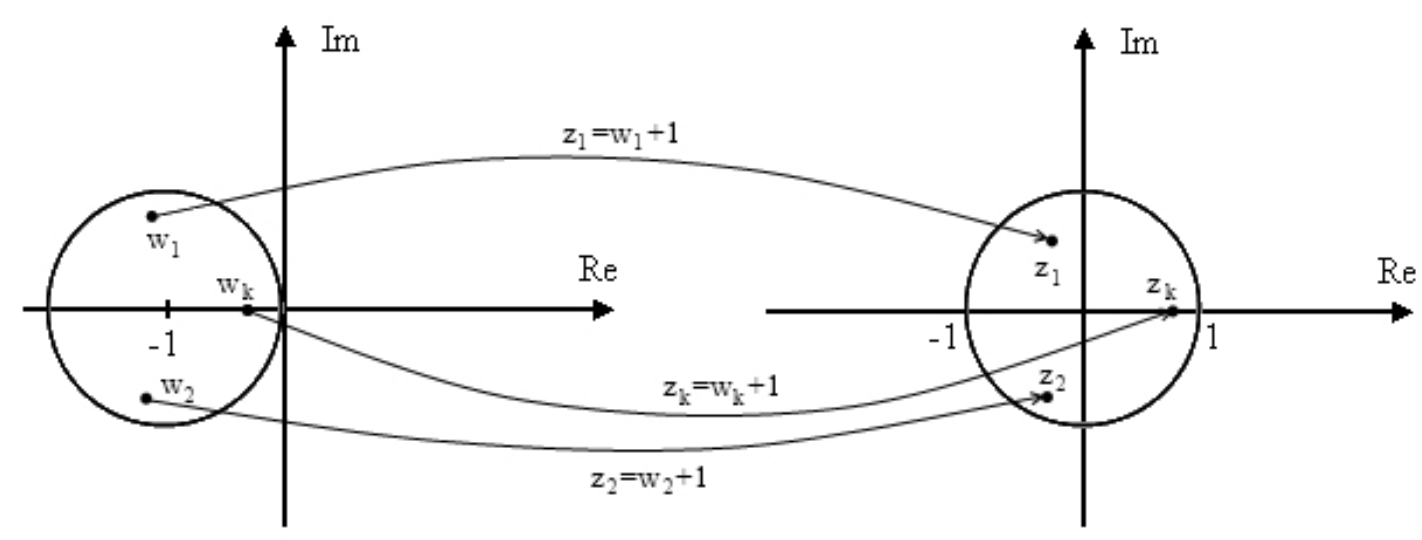

Fig. 1. Shifting the zeros $w$ into the unit circle of the complex plane.

The system (1) is called asymptotically stable if

$$
\lim _{t \rightarrow \infty, i \rightarrow \infty} x(t, i)=0 .
$$

Theorem 2. (Kaczorek, 2002) The linear continuousdiscrete $2 D$ system (1) is asymptotically stable if and only if the zeros of the polynomial

$$
\begin{aligned}
\operatorname{det}[ & \left.I_{n} s z-A_{0}-A_{1} s-A_{2} z\right] \\
= & s^{n} z^{n}+a_{n, n-1} s^{n} z^{n-1}+a_{n-1, n} s^{n-1} z^{n} \\
& +\cdots+a_{10} s+a_{01} z+a_{00}
\end{aligned}
$$

are located in the left half of the complex plane $s$ and in the unit circle of the complex plane $z$.

Theorem 3. (Kaczorek, 2002) The positive linear system

$$
\dot{x}=A x, \quad A \in M_{n}
$$

is asymptotically stable if and only if the characteristic polynomial

$$
\operatorname{det}\left[I_{n} s-A\right]=s^{n}+a_{n-1} s^{n-1}+\cdots+a_{1} s+a_{0}
$$

has positive coefficients, i.e., $a_{k}>0$ for $k=0,1, \ldots$, $n-1$.

Lemma 1. (Farina and Rinaldi, 2000) A nonnegative matrix $A \in \mathbb{R}_{+}^{n \times n}$ is asymptotically stable (a nonnegative Schur matrix) if and only if the Metzler matrix $A-I_{n}$ is asymptotically stable (a Metzler Hurwitz matrix).

\section{Main result}

Theorem 4. The positive linear continuous-discrete $2 D$ system (1) is asymptotically stable if and only if all coefficients of the polynomial

$$
\begin{aligned}
\operatorname{det} & {\left[I_{n} s(z+1)-A_{0}-A_{1} s-A_{2}(z+1)\right] } \\
= & s^{n} z^{n}+\bar{a}_{n, n-1} s^{n} z^{n-1}+\bar{a}_{n-1, n} s^{n-1} z^{n} \\
& +\cdots+\bar{a}_{10} s+\bar{a}_{01} z+\bar{a}_{00}
\end{aligned}
$$

are positive, i.e.,

$$
\bar{a}_{k, l}>0 \quad \text { for } \quad k, l=0,1, \ldots, n \quad\left(\bar{a}_{n, n}=1\right) .
$$

Proof. It is well known that the zeros $w_{1}, \ldots, w_{n}$ of the characteristic polynomial

$$
\operatorname{det}\left[I_{n} w-A\right]=w^{n}+a_{n-1} w^{n-1}+\cdots+a_{1} w+a_{0}
$$

located in the unit circle in the left half of the complex plane $w$ can be shifted into the unit circle of the complex plane $z$ by the substitution $w=z+14$ (Fig. 1), i.e., the zeros $z_{1}, \ldots, z_{n}\left(z_{k}=w_{k}+1, k=1, \ldots, n\right)$ of the characteristic polynomial

$$
\begin{aligned}
& \operatorname{det}\left[I_{n}(z+1)-A\right] \\
& \quad=z^{n}+\hat{a}_{n-1} z^{n-1}+\cdots+\hat{a}_{1} z+\hat{a}_{0} .
\end{aligned}
$$

are located in the unit circle of the complex plane.

Note that the polynomial (7) is the characteristic polynomial of the positive system

$$
\begin{aligned}
\dot{x}( & t, i+1) \\
= & \left(A_{0}+A_{2}\right) x(t, i)+\left(A_{1}-I_{n}\right) \dot{x}(t, i) \\
& +A_{2} x(t, i+1) .
\end{aligned}
$$

and its matrices $\left(A_{0}+A_{2}\right),\left(A_{1}-I_{n}\right), A_{2}$ are Metzger matrices. The sum of those matrices is also a Metzler matrix. Therefore, by Theorem 3 and the results of Kaczorek (2009), the positive continuous-discrete 2D system (1) is asymptotically stable if and only if the coefficients of the polynomial (7) are positive.

Example 1. Consider the system (1) with the matrices

$$
\begin{aligned}
& A_{0}=\left[\begin{array}{cc}
0.2 & 0 \\
0.1 & 0.1
\end{array}\right], \\
& A_{1}=\left[\begin{array}{cc}
0.4 & 0 \\
0.5 & 0.3
\end{array}\right], \\
& A_{2}=\left[\begin{array}{cc}
-0.3 & 0 \\
1 & -0.2
\end{array}\right] .
\end{aligned}
$$


The matrices (11) satisfy the conditions (3) since

$$
A_{0}+A_{1} A_{2}=\left[\begin{array}{cc}
0.08 & 0 \\
0.25 & 0.04
\end{array}\right] \in \mathbb{R}_{+}^{2 \times 2},
$$

and then the system is positive.

In this case, the polynomial (7) has the form

$$
\begin{aligned}
& \operatorname{det}\left[I_{n} s(z+1)-A_{0}-A_{1} s-A_{2}(z+1)\right] \\
& =\operatorname{det}\left[\begin{array}{c}
s(z+1)-0.2-0.4 s+0.3(z+1) \\
-0.1-0.5 s-(z+1) \\
0 \\
s(z+1)-0.1-0.3 s+0.2(z+1)
\end{array}\right] \\
& =s^{2} z^{2}+1.3 s^{2} z+0.5 s z^{2}+0.42 s^{2}+0.06 z^{2} \\
& \quad+0.53 s z+0.13 s+0.05 z+0.01 .
\end{aligned}
$$

All coefficients of the polynomial (13) are positive. Therefore, by Theorem 4, the positive continuous-discrete system (1) with (11) is asymptotically stable.

Theorem 5. The positive continuous-discrete $2 D$ linear system (1) is unstable if one of the following conditions is satisfied:

(i) $\operatorname{det}\left[-\left(A_{0}+A_{2}\right)\right] \leq 0$,

(ii) $\operatorname{det}\left[-A_{2}\right] \leq 0$,

(iii) $\operatorname{det}\left[I_{n}-A_{1}\right] \leq 0$.

Proof. Substitution of $s=z=0$ into (7) yields

$$
\operatorname{det}\left[-\left(A_{0}+A_{2}\right)\right]=\bar{a}_{00} .
$$

If the condition (i) is satisfied, then from (14) we have $\bar{a}_{00} \leq 0$, and by Theorem 4 the system (1) is unstable. Substituting $s=0$ into (7) we obtain

$$
\begin{aligned}
\operatorname{det}\left[-A_{2} z-\left(A_{0}\right.\right. & \left.\left.+A_{2}\right)\right] \\
& =\bar{a}_{0, n} z^{n}+\cdots+\bar{a}_{01} z+\bar{a}_{00},
\end{aligned}
$$

and $\operatorname{det}\left[-A_{2}\right]=\bar{a}_{0, n}$. If the condition (ii) is met, then $\bar{a}_{0 n} \leq 0$, and by Theorem 4 the system (1) is unstable. Similarly, substituting $z=0$ into (7) we obtain

$$
\begin{aligned}
\operatorname{det}\left[\left(I_{n}-A_{1}\right) s-\right. & \left.\left(A_{0}+A_{2}\right)\right] \\
& =\bar{a}_{n, 0} s^{n}+\cdots+\bar{a}_{10} s+\bar{a}_{00}
\end{aligned}
$$

and $\operatorname{det}\left[\left(I_{n}-A_{1}\right)\right]=\bar{a}_{n, 0}$. If the condition (iii) is met then $\bar{a}_{n, 0} \leq 0$ and, by Theorem 4 , the system (1) is unstable.

Example 2. Consider the system (1) with the matrices

$$
\begin{aligned}
A_{0} & =\left[\begin{array}{ll}
0.5 & 0.3 \\
0.4 & 0.4
\end{array}\right], \\
A_{1} & =\left[\begin{array}{ll}
0.2 & 0.1 \\
0.1 & 0.3
\end{array}\right], \\
A_{2} & =\left[\begin{array}{cc}
-0.3 & 0.1 \\
0.2 & -0.4
\end{array}\right] .
\end{aligned}
$$

The matrices (17) satisfy the conditions (3) since

$$
A_{0}+A_{1} A_{2}=\left[\begin{array}{ll}
0.46 & 0.28 \\
0.43 & 0.29
\end{array}\right] \in \mathbb{R}_{+}^{2 \times 2},
$$

and then the system is positive.

Using (17), we obtain

$$
\begin{aligned}
\operatorname{det}\left[-\left(A_{0}+A_{2}\right)\right] & =\operatorname{det}\left[\begin{array}{cc}
-0.2 & -0.4 \\
-0.6 & 0
\end{array}\right]=-0.24, \\
\operatorname{det}\left[-A_{2}\right] & =\operatorname{det}\left[\begin{array}{cc}
0.3 & -0.1 \\
-0.2 & 0.4
\end{array}\right]=0.1 \\
\operatorname{det}\left[I_{n}-A_{1}\right] & =\operatorname{det}\left[\begin{array}{cc}
0.8 & -0.1 \\
-0.1 & 0.7
\end{array}\right]=0.55,
\end{aligned}
$$

and the condition (i) of Theorem 5 is satisfied. Therefore, the positive system (1) with (17) is unstable.

In this case the polynomial (7) has the form

$$
\begin{aligned}
& \operatorname{det} {\left[I_{n} s(z+1)-A_{0}-A_{1} s-A_{2}(z+1)\right] } \\
&=\operatorname{det}\left[\begin{array}{cc}
s z+0.8 s+0.3 z-0.2 & -0.1 s-0.1 z-0.4 \\
-0.1 s-0.2 z-0.6 & s z+0.7 s+0.4 z
\end{array}\right] \\
&=s^{2} z^{2}+1.5 s^{2} z+0.7 s z^{2}+0.55 s^{2}+0.1 z^{2}+0.3 s z \\
& \quad-0.24 s-0.22 z-0.24,
\end{aligned}
$$

and, by Theorem 4, the system is also unstable.

\section{Concluding remarks}

New necessary and sufficient conditions for the asymptotic stability of continuous-discrete $2 \mathrm{D}$ linear systems have been established (Theorem 4). Some necessary conditions for asymptotic stability have also been given. The effectiveness of the new stability tests have been demonstrated on numerical examples. The deliberations can be also extended to fractional positive $2 \mathrm{D}$ continuous-discrete linear systems.

\section{Acknowledgment}

The author wishes to thank Professor M. Busłowicz for his valuable comments and suggestions.

This work was supported by the Ministry of Science and Higher Education in Poland under the grant no. S/WE/1/11.

\section{References}

Bistritz, Y. (2003). A stability test for continuous-discrete bivariate polynomials, Proceedings of the International Symposium on Circuits and Systems, Vol. 3, pp. 682-685.

Busłowicz, M. (2010a). Stability and robust stability conditions for a general model of scalar continuous-discrete linear systems, Pomiary, Automatyka, Kontrola 56(2): 133-135. 
Busłowicz, M. (2010b). Robust stability of the new general 2D model of a class of continuous-discrete linear systems, Bulletin of the Polish Academy of Sciences: Technical Sciences 58(4): 561-566.

Busłowicz, M. (2011). Improved stability and robust stability conditions for a general model of scalar continuousdiscrete linear systems, Pomiary, Automatyka, Kontrola 57(2): 188-189.

Dymkov, M., Gaishun, I., Rogers, E., Gałkowski, K. and Owens, D.H. (2004). Control theory for a class of 2D continuousdiscrete linear systems, International Journal of Control 77 (9): 847-860.

Farina, L. and Rinaldi, S. (2000). Positive Linear Systems: Theory and Applications, J. Wiley, New York, NY.

Gałkowski, K., Rogers, E., Paszke, W. and Owens, D.H. (2003). Linear repetitive process control theory applied to a physical example, International Journal of Applied Mathematics and Computer Science 13 (1): 87-99.

Kaczorek, T. (1998). Reachability and minimum energy control of positive 2D continuous-discrete systems, Bulletin of the Polish Academy of Sciences: Technical Sciences 46 (1): 85-93.

Kaczorek, T. (2002). Positive $1 D$ and $2 D$ Systems, SpringerVerlag, London.

Kaczorek, T. (2007). Positive 2D hybrid linear systems, Bulletin of the Polish Academy of Sciences: Technical Sciences 55(4): 351-358.

Kaczorek, T. (2008a). Positive fractional 2D hybrid linear systems, Bulletin of the Polish Academy of Sciences: Technical Sciences 56 (3): 273-277.

Kaczorek, T. (2008b). Realization problem for positive 2D hybrid systems, COMPEL 27 (3): 613-623.

Kaczorek, T. (2009). Stability of positive continuous-time linear systems with delays, Bulletin of the Polish Academy of Sciences: Technical Sciences 5744: 395-398.

Kaczorek, T., Marchenko, V. and Sajewski, Ł. (2008). Solvability of 2D hybrid linear systems-Comparison of the different methods, Acta Mechanica et Automatica 2(2): 59-66.
Sajewski, Ł. (2009). Solution of 2D singular hybrid linear systems, Kybernetes 38 (7/8): 1079-1092.

Xiao, Y. (2001a). Stability test for 2-D continuous-discrete systems, Proceedings of the 40th IEEE Conference on Decision and Control, Orlando, FL, USA, Vol. 4, pp. 36493654 .

Xiao, Y. (2001b). Robust Hurwitz-Schur stability conditions of polytopes of 2-D polynomials, Proceedings of the 40th IEEE Conference on Decision and Control, Orlando, FL, USA, Vol. 4, pp. 3643-3648.

Xiao, Y. (2003). Stability, controllability and observability of 2-D continuous-discrete systems, Proceedings of the International Symposium on Circuits and Systems, Bangkok, Thailand, Vol. 4, pp. 468-471.

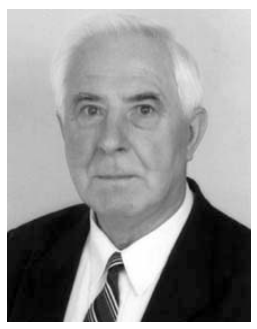

Tadeusz Kaczorek received the M.Sc., Ph.D. and D.Sc. degrees in electrical engineering from the Warsaw University of Technology in 1956, 1962 and 1964, respectively. In the years 1968-69 he was the dean of the Electrical Engineering Faculty, and in the period of 1970-73 he was a deputy rector of the Warsaw University of Technology. In 1971 he became a professor and in 1974 a full professor at the same university. Since 2003 he has been a professor at Białystok Technical University. In 1986 he was elected a corresponding member and in 1996 a full member of the Polish Academy of Sciences. In the years 1988-1991 he was the director of the Research Centre of the Polish Academy of Sciences in Rome. In 2004 he was elected an honorary member of the Hungarian Academy of Sciences. He has been granted honorary doctorates by several universities. His research interests cover the theory of systems and automatic control systems theory, especially singular multidimensional systems, positive multidimensional systems, and singular positive 1D and 2D systems. He initiated research in the field of singular 2D and positive 2D systems. He has published 24 books (six in English) and over 950 scientific papers. He has also supervised 69 $\mathrm{Ph} . \mathrm{D}$. theses. He is the editor-in-chief of the Bulletin of the Polish Academy of Sciences: Technical Sciences and a member of editorial boards of ten international journals.

Received: 24 January 2011 Revised: 9 March 2011 